\title{
Comparison of Full Wavefield Synthetics with Frequency-Dependent Traveltimes Calculated Using Wavelength-Dependent Velocity Smoothing
}

\author{
Jianxiong Chen and Colin A. Zelt \\ Department of Earth Science, Rice University, Houston, TX 77005 \\ Email: jianxiong.chen.rice@gmail.com; czelt@rice.edu
}

\begin{abstract}
Ray theory-based traveltime calculation that assumes infinitely high frequency wave propagation is likely to be invalid in the near-surface (upper tens of meters) due to the relatively large seismic wavelength compared with the total travel path lengths and the scale of the nearsurface velocity heterogeneities. The wavelength-dependent velocity smoothing (WDVS) algorithm calculates a frequency-dependent, first-arrival traveltime by assuming that using a wavelength-smoothed velocity model and conventional ray theory is equivalent to using the original unsmoothed model and a frequency-dependent calculation. This paper presents comparisons of WDVS-calculated traveltimes with band-limited full wavefield synthetics including the results from 1) different velocity models, 2) different frequency spectra, 3) different values of a free parameter in the WDVS algorithm, and 4) different levels of added noise to the synthetics. The results show that WDVS calculates frequency-dependent traveltimes that are generally consistent with the first arrivals from band-limited full wavefield synthetics. Compared to infinite-frequency traveltimes calculated using conventional ray theory, the WDVS frequency-dependent traveltimes are more consistent with the first arrivals picked from full wavefield synthetics in terms of absolute time and trace-to-trace variation. The results support the use of WDVS as the forward modeling component of a tomographic inversion method, or any seismic method that involves modeling first-arrival traveltimes.
\end{abstract}

\section{Introduction}

Ray theory-based traveltime calculation (e.g., Vidale, 1988) and ray tracing (e.g., Julian and Gubbins, 1977; Um and Thurber, 1987) are efficient methods to approximately simulate seismic wave propagation assuming high frequencies (equivalently small wavelengths), that can serve as the basis for seismic tomography (e.g., Zelt and Barton, 1998) and seismic migration (e.g., Gray and May, 1994). Compared to global- and crustal-scale studies, the high-frequency assumption is likely to be less valid, and the resulting effects more significant in near-surface seismic studies (upper tens of meters) due to the relatively large seismic wavelength compared with the total travel path lengths and the scale of near-surface velocity heterogeneities (e.g., Gao et al., 2007). This motivates the desire to model the finite-frequency behavior of wave propagation within the cost-effective framework of traveltime calculation or ray tracing, without solving the wave equation.
Zelt and Chen (2016) present a frequency-dependent traveltime tomography method to invert first arrivals for seismic velocity estimation, whose key feature is calculating frequency-dependent traveltimes using wavelength-dependent velocity smoothing (WDVS) for forward modeling. WDVS is implemented in a wavefront-tracking algorithm (Vidale, 1988, 1990) but is based on the wavelength-smoothing technique applied by Lomax (1994) in the context of ray tracing. Both WDVS and the wavelength-smoothing technique are designed to simulate the frequency-dependent behavior of finite-frequency wave propagation, although they are not formally derived from wave or ray theory. Zelt and Chen (2016) focus on presenting the calculation method of frequency-dependent traveltimes and their use in tomographic inversion, including sensitivity kernels based on WDVS, for improved velocity estimation with applications to synthetic and real data. This paper focuses on justifying the use of WDVS to effectively model frequency-dependent traveltimes through comparisons with band-limited full wavefield synthetics. 
Journal of Environmental and Engineering Geophysics

Similar comparisons have been done by other researchers to justify their frequency-dependent traveltimes either produced from a traveltime calculation algorithm (e.g., Biondi, 1997; Hogan and Margrave, 2007) or resulting from a ray tracing algorithm (e.g., Washbourne et. al., 2008; Protosov et al., 2011; Yarman et al., 2013; Chen et al., 2014). In these studies the calculated traveltimes are compared with full wavefield synthetics by either displaying the calculated traveltimes on wavefield snapshots for a visual comparison without explicitly picking the traveltimes from the wavefield snapshots (Biondi, 1997; Hogan and Margrave, 2007; Yarman et al., 2013; Chen et al., 2014), or by displaying the calculated traveltimes on seismograms for a visual comparison with (Washbourne et. al., 2008) or without (Protosov et al., 2011) explicitly picking the first arrivals from the seismograms. In this paper the calculated traveltimes are compared with both the synthetic seismograms and the picked first arrivals from the synthetics (representing the "true" traveltimes).

The purpose of this paper is to validate the use of WDVS as the forward modeling component of traveltime tomography or for other seismic methods that involve modeling first-arrival traveltimes. Comparisons of the WDVS traveltimes with the synthetics include results from 1) different velocity models, 2) different frequency spectra, 3) different values of a free parameter in the WDVS algorithm, and 4) different levels of added noise to the synthetics.

\section{Wavelength-Dependent Velocity Smoothing}

This section summarizes the key steps of implementing WDVS in a wavefront-tracking algorithm to calculate a frequency-dependent traveltime; the reader is referred to Zelt and Chen (2016) for a complete description of the background, motivation, and implementation of the WDVS algorithm including examples illustrating the frequency-dependent behavior of the traveltimes and travel paths.

WDVS is based on the wavelength-smoothing technique that models finite-frequency wave propagation within the context of ray tracing (Lomax, 1994). Figure 1 illustrates the expected behavior of WDVS in the context of ray/wave paths. A wave path represents the travel path of finite-frequency wave propagation, i.e., a frequency-dependent ray path. WDVS and the wavelength-smoothing technique of Lomax (1994) assume that a wave path will be more influenced by heterogeneities closer to the path than those farther away from the path, and the extent of sensitivity should be about

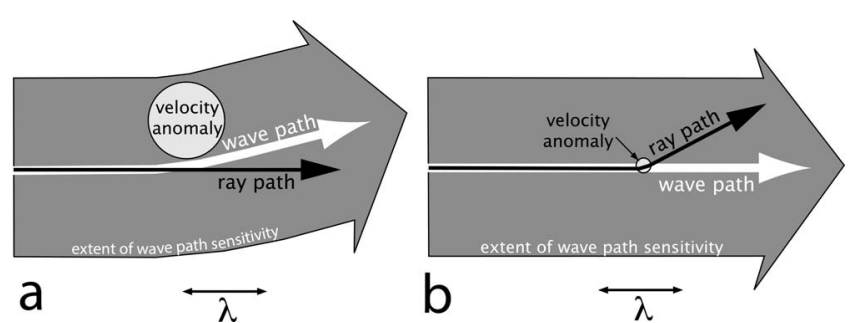

Figure 1. Cartoon showing different behavior of ray paths and wave paths (from Zelt and Chen, 2016). a) $A$ ray path is unperturbed but a wave path is deflected by a wavelength-scale velocity anomaly that is within one wavelength of the path. b) A ray path is strongly perturbed but a wave path is not affected by a small velocity anomaly on the path. The wavelength-dependent velocity smoothing (WDVS) algorithm assumes the extent of the wave path sensitivity to velocity heterogeneities is determined by the local seismic wavelength.

one wavelength of the seismic wave, while a ray path representing infinitely high frequency wave propagation is only sensitive to heterogeneities on the path. As a result, the ray and wave paths can behave significantly different when the size of the velocity anomaly is comparable (Fig. 1(a)) or smaller (Fig. 1(b)) than the wavelength, which are likely the cases in the nearsurface. Therefore, WDVS should be used in any traveltime tomographic study where high-resolution is desired. For models without anomalies comparable in size to the wavelength, such as uniform or layered models, WDVS will have little or no effect.

WDVS is implemented in two steps. First, a smoothing of the velocity model is conducted according to the local seismic wavelength using a cosine-squared weighting function with a geometrical correction factor specific to 2-D and 3-D models. Then, the smoothed model is used in a conventional ray theory-based, wavefront-tracking algorithm (eikonal solver) under the assumption that the traveltime in the smoothed model is equivalent to the traveltime that would be calculated using a frequency-dependent simulation in the original unsmoothed model. Zelt and Chen (2016) use the 2-D (Vidale, 1988) and 3-D (Vidale, 1990) wavefronttracking algorithms, with modifications to account for large velocity gradients (Hole and Zelt, 1995), to solve the eikonal equation for traveltimes on a fine square grid using finite-difference operators, with the velocity model specified at each node of the grid.

Figure 2 shows examples of the velocity smoothing weights applied by WDVS for a single node in 2-D and $3-\mathrm{D}$ versions of a linear velocity gradient. The frequency $(200 \mathrm{~Hz})$ and the velocity gradient are realistic for the 

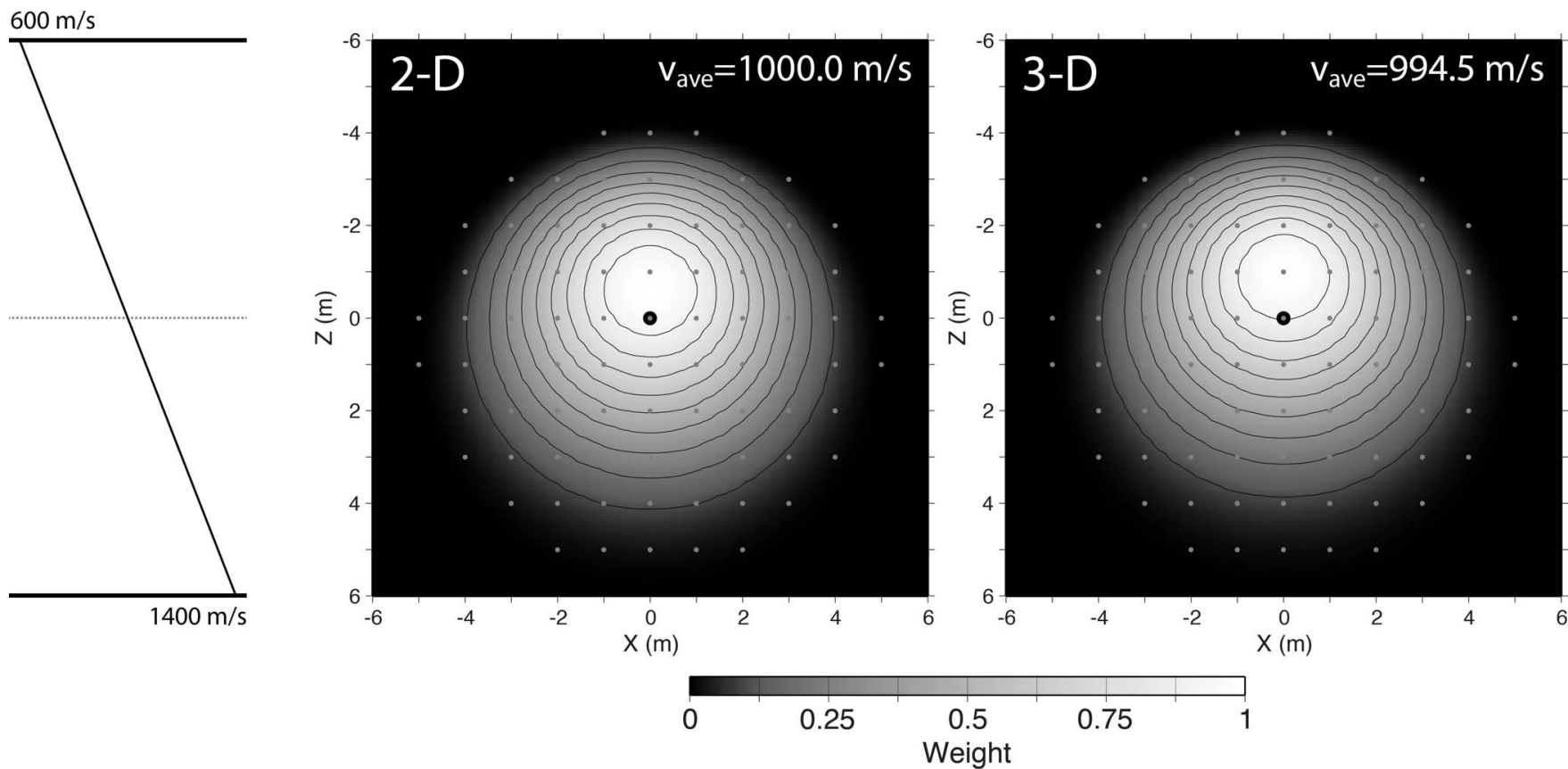

Figure 2. Examples of velocity smoothing weights applied by WDVS at $200 \mathrm{~Hz}$ for a single node (black circle) in 2-D and 3-D versions of a linear velocity gradient (modified after Zelt and Chen, 2016). For the 3-D model, weights are only shown in the vertical plane in which the central node (black circle) is located. The central node is located where it is expected to have an average velocity of $1,000 \mathrm{~m} / \mathrm{s}$ (horizontal dashed line in the velocity profile on the left). The calculated $V_{\text {ave }}$, based on the smoothing weights and the unsmoothed model, is indicated. A uniform grid with a $0.05 \mathrm{~m}$ node spacing is used; gray dots indicate every 10th node in the grid in the horizontal and vertical directions. Contour interval is 0.1 . The slight difference between the weights is due to a geometrical correction term described in the text.

near-surface. All nodes within one period of the central node, extending outward in all directions in 2-D or 3-D from it have a non-zero weight. At each node, the weight is a product of the cosine-squared weighting function and a geometrical correction term. The cosine-squared weighting function has a maximum value of one at the central node, decreasing to zero at one period away from the center. Thus, the average velocity for a single node, $\bar{v}$, is

$$
\bar{v}=\frac{\sum_{i=1}^{N} \frac{\cos ^{2}\left(\frac{\pi}{2} \frac{t}{T}\right)}{v_{i}^{p}} v_{i}}{\sum_{i=1}^{N} \frac{\cos ^{2}\left(\frac{\pi}{2} \frac{t}{T}\right)}{v_{i}^{p}}}
$$

where $N$ is the number of nodes within one period of the central node, $t$ is the straight-line traveltime between the central node and the node with velocity $v_{i}, T$ is the period corresponding to the frequency used, and $p=2$ or 3 for 2-D or 3-D models, respectively (Zelt and Chen, 2016). The $1 / v^{p}$ geometrical correction term accounts for the fact that one wavelength from the central node is longer and includes more velocity nodes in the higher velocity regions than in the lower velocity regions. Without this correction the higher velocity nodes will be overrepresented. For both the 2-D and 3-D cases in Fig. 2, the smoothed velocity for the central node should be $1,000 \mathrm{~m} / \mathrm{s}$. The $3-\mathrm{D}$ case has a $0.6 \%$ error due to the imprecision of the geometrical term when using a discretized model.

As Zelt and Chen (2016) point out, the Vidale algorithms calculate traveltimes corresponding to a frequency whose wavelength is on the order of the model node spacing, and normally the grid is fine enough so that these traveltimes are effectively infinitely high frequency traveltimes. In the rest of this paper, the traveltimes calculated using an unsmoothed model are referred to as infinite-frequency traveltimes.

\section{Experiment Setup}

A time-domain finite-difference acoustic wave simulator (Symes et al., 2011) generates the synthetics using a plane wave composed of two-excursion keuper wavelets (Brenders and Pratt, 2007). The plane wave travels vertically through a $2-\mathrm{D}$ velocity model contain- 

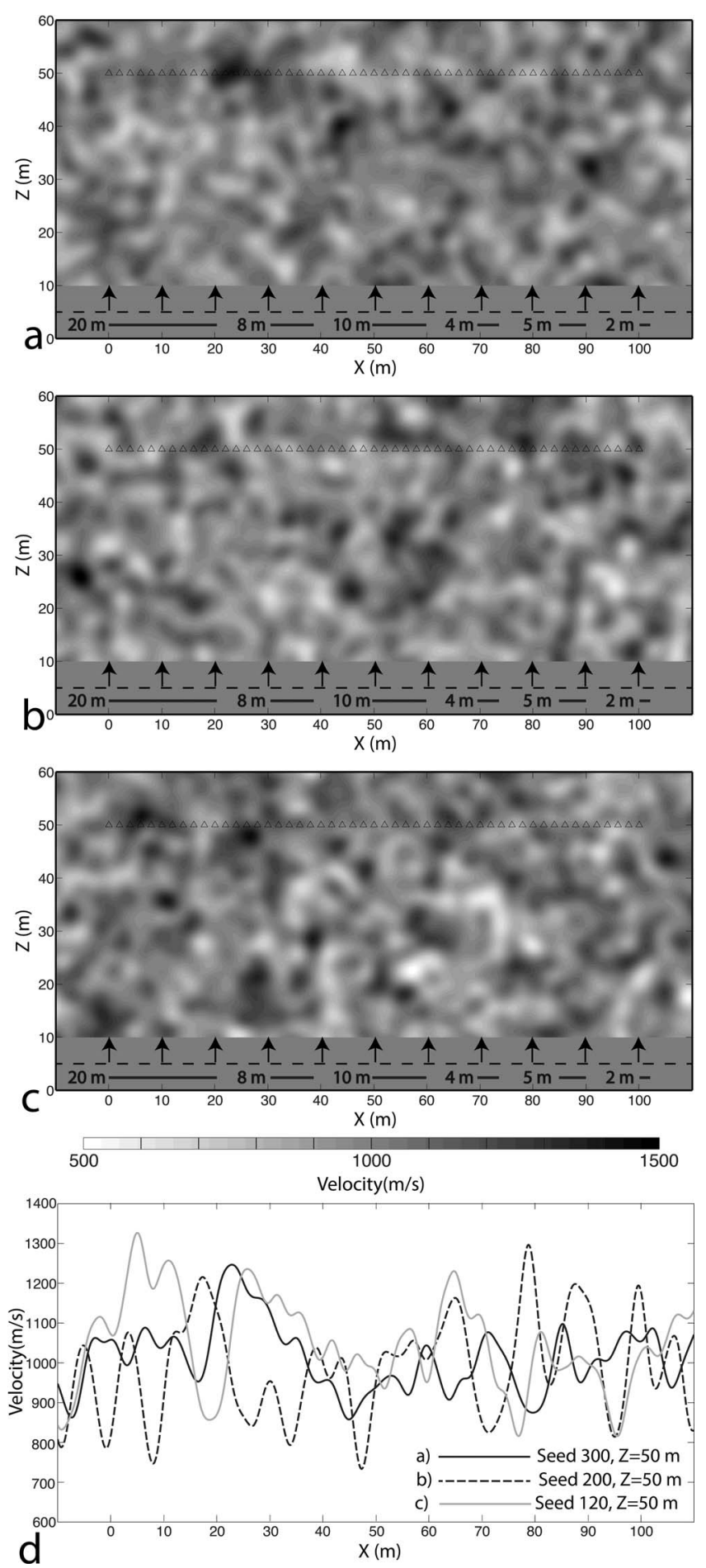

Figure 3. Velocity models with randomly distributed velocity perturbations up to $\pm 50 \%$ from the constant background model $(1,000 \mathrm{~m} / \mathrm{s})$. The three models were produced using different seed numbers for initializing a random number generator. (a) Seed300 model has an RMS perturbation of $113 \mathrm{~m} / \mathrm{s}$ from the constant background model. (b) Seed-200 model has an RMS perturbation of $120 \mathrm{~m} / \mathrm{s}$. (c) Seed-120 model has an RMS perturbation of $131 \mathrm{~m} / \mathrm{s}$. (d) ing randomly distributed velocity anomalies, and is sampled by a horizontal array of 50 receivers, evenlyspaced, $45 \mathrm{~m}$ away (Fig. 3). Wavelets with dominant frequencies of 50, 100, and $200 \mathrm{~Hz}$ (Fig. 4) are tested. Three random velocity models (Fig. 3) are used by adding random velocity anomalies generated by the Matlab function 'randn' using different seed numbers $(300,200,120)$ to a constant background velocity model $(1,000 \mathrm{~m} / \mathrm{s})$ and applying 200 -iterations of a $3 \times 3$ node smoothing filter. The velocity anomalies of these three models have similar length scales and magnitudes, but randomly different spatial distributions (Fig. 3(d)). For both the acoustic simulator and the wavefront-tracking eikonal solver, the velocity models are parameterized using a square grid with a node spacing of $0.1 \mathrm{~m}$. Adding random noise that has the same bandwidth as the noisefree synthetics produced the noisy synthetics. The rootmean-square (RMS) amplitude of the added noise is proportional to the root-mean-square amplitude of the early arrivals of the noise-free synthetics. The source spectra, the magnitude of the velocity anomalies, the relative size of the velocity anomalies compared to the seismic wavelengths, and the amplitudes of added noise to the synthetics are realistic for near-surface studies.

Zelt and Chen (2016) suggest two ways to pick the first arrivals of real data depending on the signal-to-noise ratio. When the data have a high signal-to-noise ratio, the onsets are picked; when the data have a low-signalto-noise ratio, the picks are based on a trace-to-trace correlation of the peaks/troughs in the first-arrival waveform and made by a certain time advance from the peaks/troughs toward the onsets that would be predicted without noise. In this paper, to avoid biases from manual picks, in the comparisons of the WDVS traveltimes with the noise-free synthetics, the onsets of the synthetics are picked based on an amplitude threshold that is $1 \%$ of the RMS amplitude of the early arrivals. In the comparisons with the WDVS traveltimes with the noisy synthetics, the first arrivals are not explicitly picked. The extent of the early arrivals is defined by a time window based on the dominant-

Velocity profiles of the three models at $z=50 \mathrm{~m}$. A horizontal plane wave source is introduced into the models at $z=5 \mathrm{~m}$ for simulating the acoustic wavefield synthetics. An array of 50 receivers with an even $2-\mathrm{m}$ spacing is positioned at $z=50 \mathrm{~m}$. The solid black lines at the bottom of each model represent the 20-, 8-, 10-, 4-, 5-, and 2-m wavelengths, corresponding to 50-, 125-, 100-, 250-, 200-, and 500$\mathrm{Hz}$ waves in the background velocity. 
Chen and Zelt: Frequency-Dependent First-Arrival Traveltime Calculation

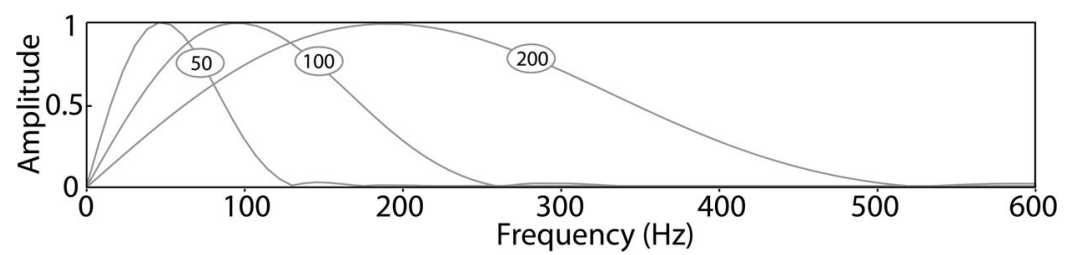

Figure 4. Source spectra of a two-excursion keuper wavelet (Brenders and Pratt, 2007) with dominant frequencies of 50, 100 and $200 \mathrm{~Hz}$ used in the acoustic wavefield modeling. For modeling frequency-dependent traveltimes using WDVS, high-end frequencies of 125,250 and $500 \mathrm{~Hz}$, respectively, are used.

offset ( $m$ )

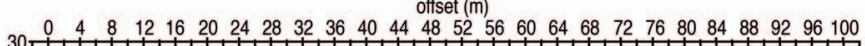

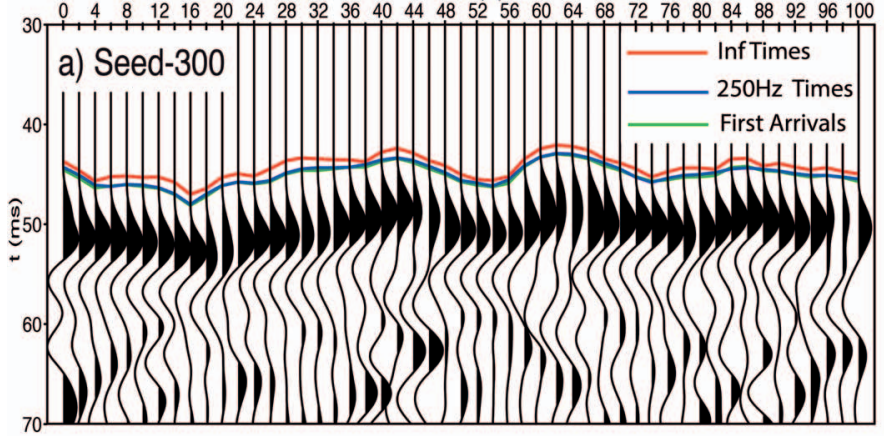

offset ( $m$ )

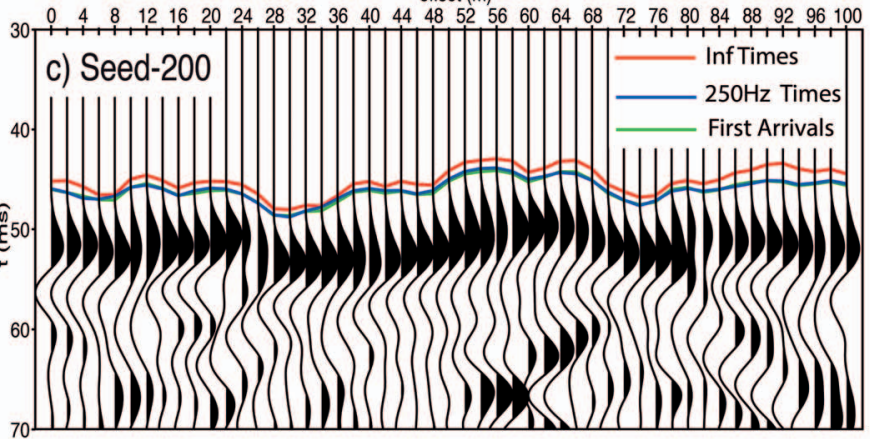

offset $(m)$

$\begin{array}{llllllllllllllllllllllllll}0 & 4 & 8 & 12 & 16 & 20 & 24 & 28 & 32 & 36 & 40 & 44 & 48 & 52 & 56 & 60 & 64 & 68 & 72 & 76 & 80 & 84 & 88 & 92 & 96 & 100\end{array}$

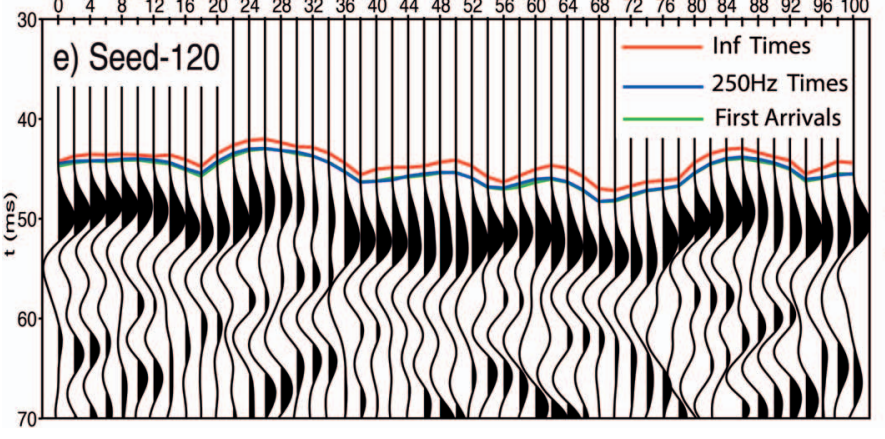

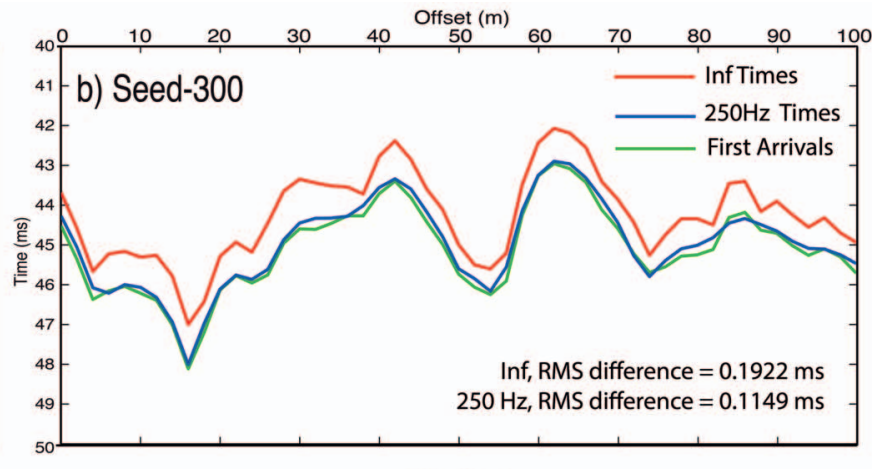
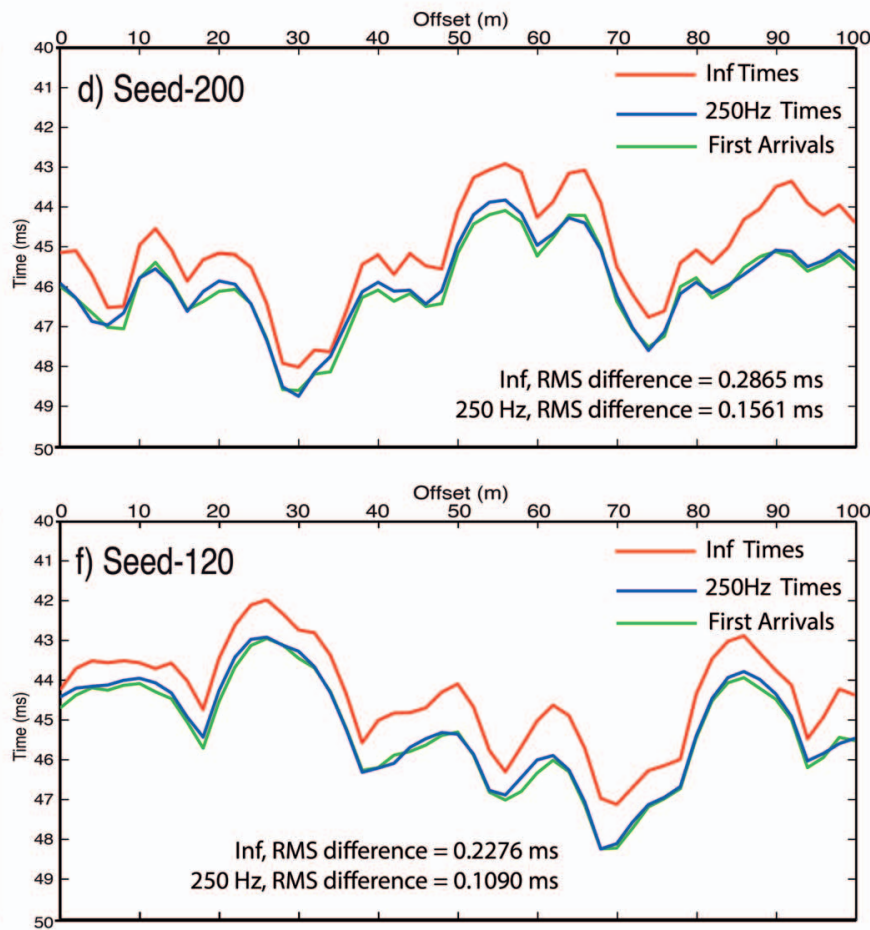

Figure 5. Comparisons of infinite-frequency traveltimes from conventional ray theory (red or grey lines) and frequency-dependent traveltimes from WDVS (blue or dashed black lines) with picked first arrivals from noisefree synthetic traces using the 3 models in Fig. 3 and the source spectra with a dominant frequency of $100 \mathrm{~Hz}$ (Fig. 4) (green or solid black lines). The RMS differences between the infinite-frequency/frequency-dependent traveltimes and the picked first arrivals from the synthetics are indicated in the figures. Subplots a), c), and e) compare the calculated traveltimes with the picked arrivals with the acoustic synthetics as the background, using Seed-300, Seed-200, Seed-120 models, respectively. Subplots b), d), and f) compare the calculated traveltimes with the picked first arrivals with an expanded vertical axis, corresponding to that in a), c), and e), respectively. 
Journal of Environmental and Engineering Geophysics
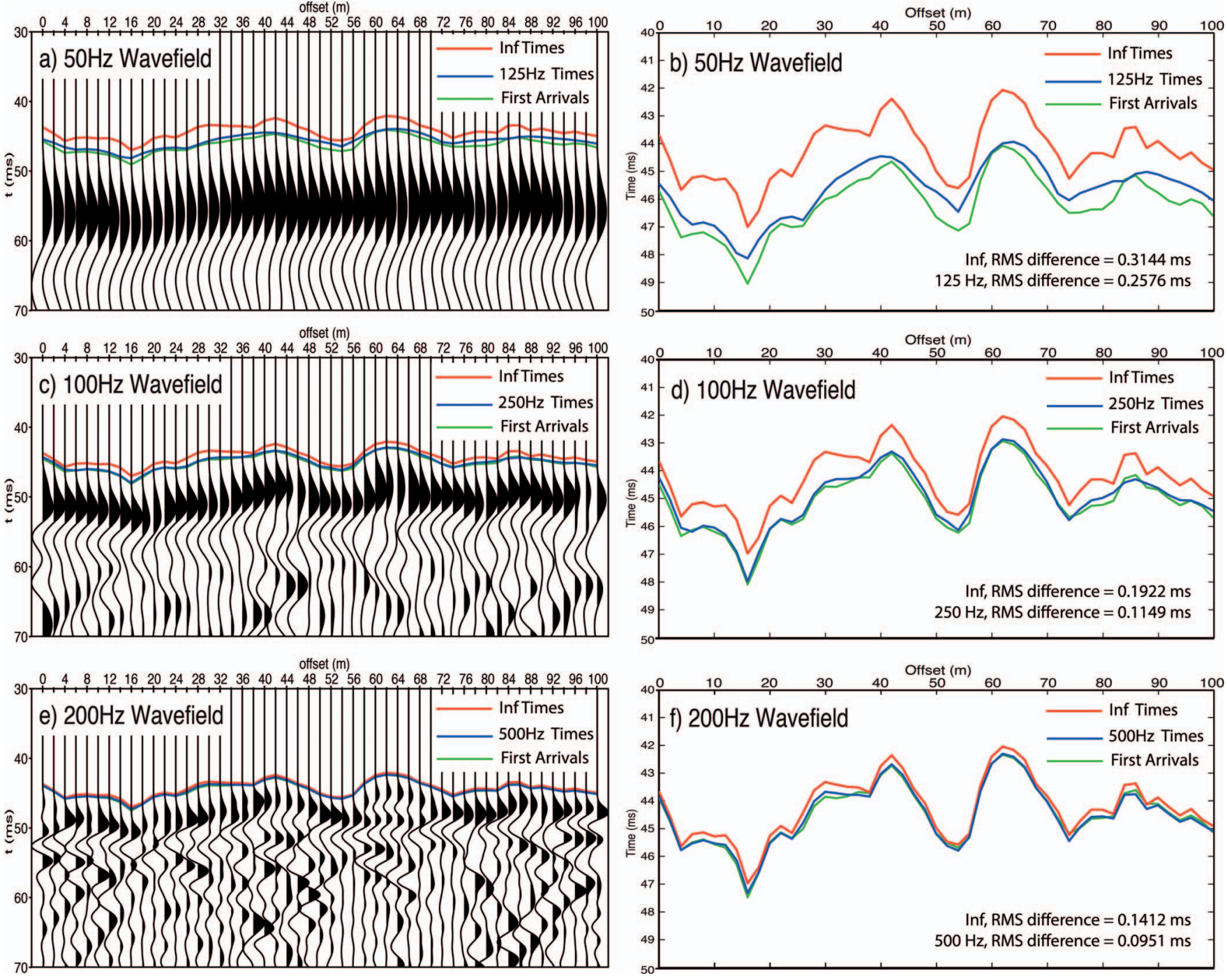

Figure 6. Comparisons of infinite-frequency traveltimes from conventional ray theory (red or grey lines) and frequency-dependent traveltimes from WDVS (blue or dashed black lines) with picked first arrivals from noisefree synthetic traces using the Seed-300 model in Fig. 3 and different source spectra in Fig. 4 (green or solid black lines). The RMS differences between the infinite-frequency/frequency-dependent traveltimes and the picked first arrivals from the synthetics are indicated in the figures. Subplots a), c), and e) compare the calculated traveltimes with the picked first arrivals with the acoustic synthetics as the background, using source wavelets with a dominant frequency of 50, 100, $200 \mathrm{~Hz}$, respectively. Subplots b), d), and f) compare the calculated traveltimes with the picked first arrivals with an expanded vertical axis, corresponding to that in a), c), e), respectively.

frequency of the wavelet that is used to generate the synthetics: 40, 20 and $10 \mathrm{~ms}$ for the 50-, 100- and 200Hz-wavelet synthetics, respectively, each starting from the calculated infinite-frequency traveltimes.

Both the infinite-frequency and frequency-dependent traveltimes are calculated for the comparisons. For using WDVS to model the first arrivals picked from band-limited real data, Zelt and Chen (2016) suggest that the selected modeling frequency should be based on the data spectra and signal-to-noise ratio: the highest frequency in the spectra is selected to model the first arrivals picked from high signal-to-noise ratio data and the dominant frequency is selected to model the first arrivals picked from low signal-to-noise ratio data. In this paper, the high-end frequencies $(125 \mathrm{~Hz}, 250 \mathrm{~Hz}$, $500 \mathrm{~Hz}$ ) of each wavelet spectra (Fig. 4) are selected for modeling the first arrivals of the noise-free synthetics and the dominant frequencies of each wavelet spectra are selected for modeling the first arrivals of the noisy synthetics.

To quantitatively compare the calculated traveltimes with the picked first arrivals from the synthetics, a 
Chen and Zelt: Frequency-Dependent First-Arrival Traveltime Calculation

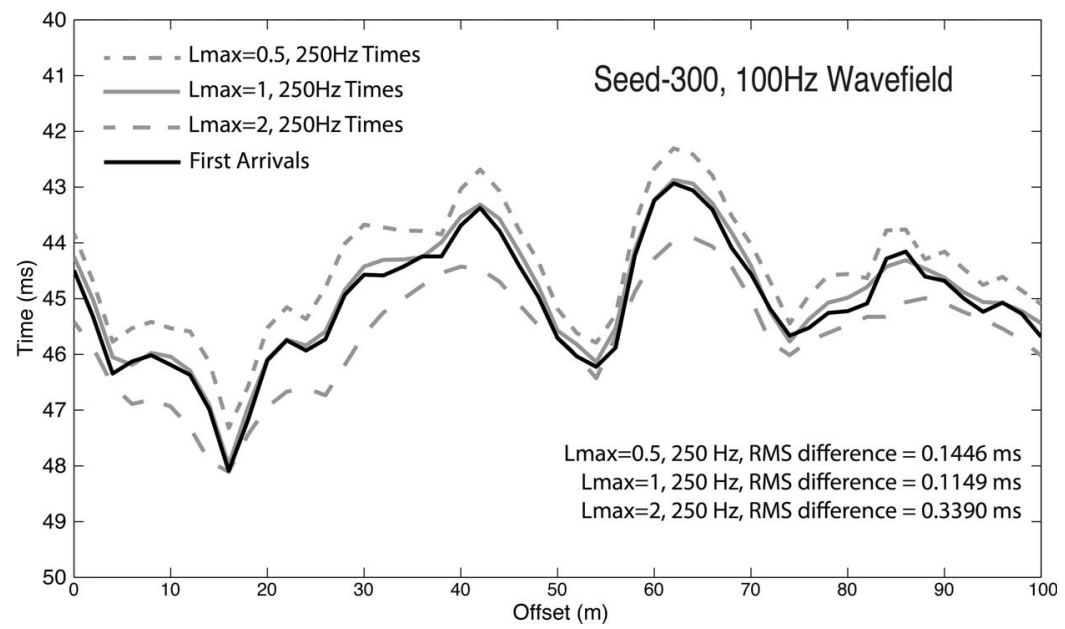

Figure 7. Comparisons of frequency-dependent traveltimes calculated from WDVS using three different $L_{\max }$ values of $0.5,1$, and 2 (gray lines), with the picked first arrivals from noise-free synthetic traces using the Seed300 model in Fig. 3 and the source spectra with a dominant frequency of $100 \mathrm{~Hz}$ in Fig. 4 (black line). The RMS differences between the frequency-dependent traveltimes and the picked first arrivals from the synthetics are indicated in the figure.

minimum RMS difference is calculated to compare two sets of traveltimes. The minimum RMS difference is calculated by bulk shifting the two sets of traveltimes by subtracting the mean of each set. As a result, the minimum RMS difference excludes the effect of a bulk time shift between the two sets of traveltimes but reflects the trace-to-trace traveltime variations.

\section{Results}

Figure 5 presents the comparisons of the infinitefrequency and frequency-dependent WDVS traveltimes with the picked first arrivals, resulting from the 3 different models (Fig. 3) and the source spectra with dominant and high-end frequencies of 100 and $250 \mathrm{~Hz}$, respectively (Fig. 4). In all cases, the $250 \mathrm{~Hz}$ WDVScalculated traveltimes are more consistent with the picked first arrivals compared to the infinite-frequency traveltimes in terms of absolute time and the trace-totrace traveltime variations as indicated by a smaller RMS difference between the frequency-dependent traveltimes and the picked traveltimes.

Figure 6 presents the comparisons of the infinitefrequency and frequency-dependent WDVS traveltimes with the picked first arrivals, resulting from three source spectra with different frequencies (Fig. 4) and the Seed300 model (Fig. 3). In each case the results show that the WDVS-calculated frequency-dependent traveltimes are more consistent with the picked first arrivals compared to the infinite-frequency traveltimes in terms of absolute time and trace-to-trace traveltime variations. The results also show the frequency dependence of both the picked first arrivals and WDVS-calculated traveltimes. As the frequency increases, the picked and frequency-dependent travel curves become rougher and approach the infinite-frequency traveltime curve. As expected, the frequency-dependent effects are less significant for higher frequencies. Also, given the characteristic length scale of the velocity anomalies in the model, the infinitefrequency, $500 \mathrm{~Hz}$ and picked traveltimes are nearly the same, as expected. Figure 6 also shows that at progressively lower frequency, there is a dispersive effect whereby the lower frequency traveltimes reflect a lower average velocity due to the fatter, low-frequency wave paths averaging over a larger portion of the model, whereas narrower, high-frequency wave paths traverse a greater proportion of high-velocity regions of the model.

Figure 7 presents a comparison of the frequencydependent WDVS traveltimes calculated using three different values of a free parameter in the WDVS algorithm, $L_{\max }$, with the picked first arrivals, resulting from the Seed-300 model (Fig. 3) and the source spectra with dominant and high-end frequencies of 100 and 250 $\mathrm{Hz}$, respectively (Fig. 4). In the WDVS algorithm, the value of $L_{\max }$ equals the number of periods from a central model node over which the velocity is averaged for that node (Zelt and Chen, 2016). The results show that among $0.5,1$, and 2 , the best $L_{\max }$ value is 1 in that its corresponding frequency-dependent traveltimes are most consistent with the picked first arrivals in terms of absolute time, and similarities in trace-to-trace traveltime variations. When the $L_{\max }$ value is 0.5 , the 


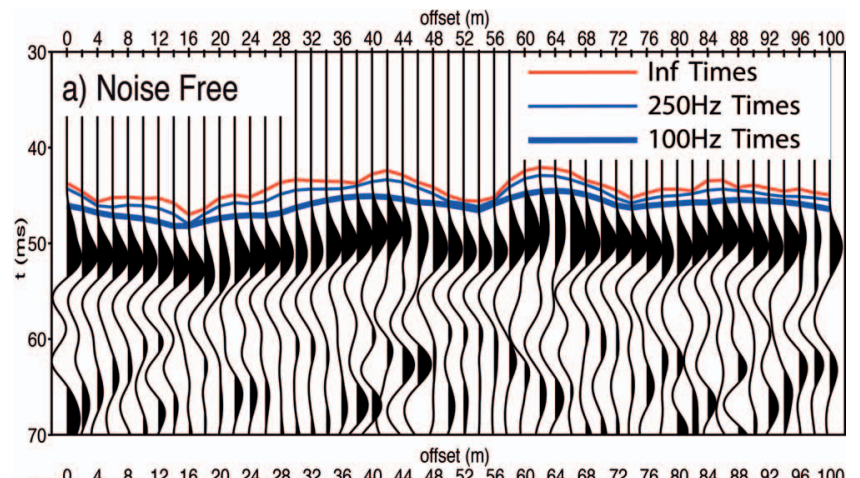

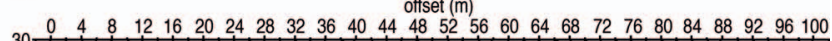

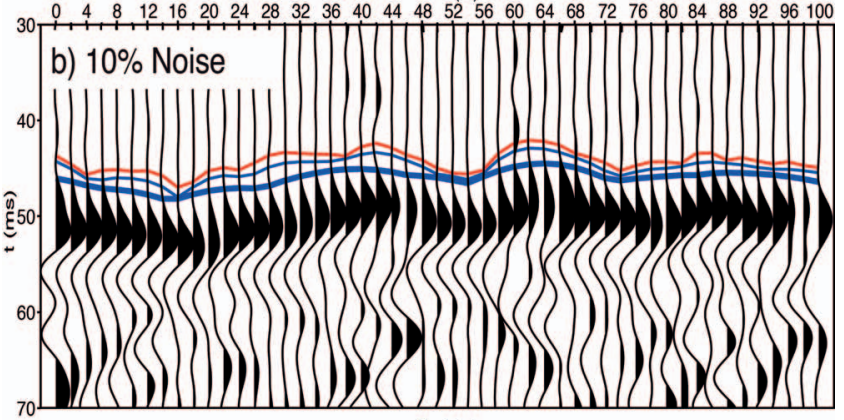

offset (m)

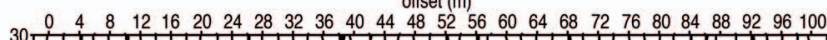

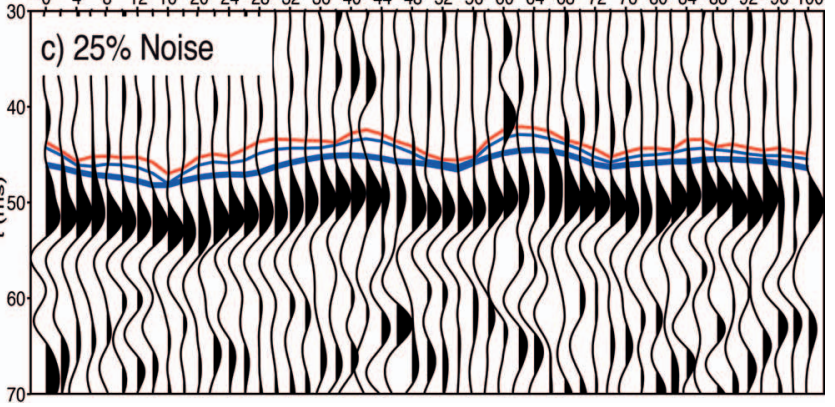

Figure 8. Comparisons of infinite-frequency traveltimes from conventional ray theory (red or grey lines) and frequency-dependent traveltimes from WDVS (blue or black lines) with the noisy acoustic synthetics using the Seed-300 model in Fig. 3 and the source spectra with a dominant frequency of $100 \mathrm{~Hz}$ in Fig. 4. (a) Noise-free synthetics. (b) $10 \%$ noise synthetics. (c) $25 \%$ noise synthetics. The random noise added to the synthetics in (b) and (c) has the same bandwidth as the synthetics and its RMS amplitude is based on the RMS amplitude of the noise-free early arrivals in a $20-\mathrm{ms}$ window after the infinite-frequency traveltime of each trace. The two sets of noise are only different in amplitude and represent the same sequence of random numbers.

frequency-dependent traveltimes are biased fast; when the $L_{\max }$ value is 2 , the frequency-dependent traveltimes are biased slow.

Figure 8 presents the comparisons of the infinitefrequency and frequency-dependent WDVS traveltimes with the noisy synthetics, resulting from the Seed-300 model (Fig. 3) and the source spectra with dominant and high-end frequencies of 100 and $250 \mathrm{~Hz}$, respectively (Fig. 4). Both 100 and $250 \mathrm{~Hz}$ WDVS traveltimes are calculated and noise-free, $10 \%$ noisy, and 25\% noisy synthetics are presented. As the noise level increases, both the infinite-frequency and high-end $(250 \mathrm{~Hz})$ frequency-dependent traveltimes seem less realistic, i.e., less likely to be consistent with the first arrivals that one would pick from the noisy traces.

\section{Discussion and Conclusions}

This paper presents a series of comparisons of WDVS-calculated traveltimes with band-limited full wavefield synthetics, showing that WDVS can calculate frequency-dependent traveltimes that are significantly more consistent with the picked first arrivals from the band-limited full wavefield synthetics than conventional ray theory infinite-frequency traveltimes. This suggests that WDVS captures some of the frequency-dependent behavior of finite-frequency wave propagation. The presented examples are realistic for near-surface seismic wave propagation in terms of the source spectra, the magnitude of the velocity anomalies, the relative size of the velocity anomalies compared to the wavelengths, and the noise level. Compared to infinite-frequency traveltimes, the WDVS-calculated traveltimes are more consistent with the picked first arrivals (considered the "true" traveltimes) in terms of absolute time and traceto-trace traveltime variations. These results justify the use of WDVS for modeling frequency-dependent traveltimes that can serve as the forward modeling component of a tomographic inversion method (Zelt and Chen, 2016).

The extra step when using WDVS to calculate a frequency-dependent traveltime, compared to using conventional ray theory, is to specify a frequency for the velocity smoothing. The tests in this paper select the frequency based on the spectra of the source wavelet and the noise level in the synthetics: the high-end frequency is selected for modeling the first arrivals of noise-free synthetics and the dominant frequency is selected for modeling the first arrivals of noisy synthetics. In dealing with real data, one should analyze the spectra of the early arrivals and the level of noise to select a proper frequency using the results presented here for synthetic data as a guideline (e.g., Zelt and Chen, 2016; Chen et al., 2016).

Adjusting the $L_{\max }$ value (Fig. 7) has the same effect as varying the modeling frequency. Using an $L_{\max }$ value smaller than 1 is equivalent to using a proportion- 


\section{Chen and Zelt: Frequency-Dependent First-Arrival Traveltime Calculation}

ately higher frequency and using an $L_{\max }$ value larger than 1 is equivalent to using a proportionately lower frequency. The results presented here suggest that using an $L_{\max }$ value of 1 means that the appropriate modeling frequencies for high and low signal-to-noise ratio data are the high-end and dominant frequencies, respectively.

The WDVS algorithm, including its use in frequency-dependent traveltime tomography (Zelt and Chen, 2016), is most applicable to near-surface studies of P-and S-wave first-arrival-time data where the typical seismic wavelength is large relative to the total path lengths and the length-scale of velocity anomalies (e.g., Chen et al., 2016; Chen and Zelt, 2016). WDVS is also applicable to exploration- and crustal-scale data, but the effects will be less significant (e.g., Kiser et al., 2016). WDVS differs from several other frequency-dependent, ray-based approaches (e.g., Spetzler and Snieder, 2004), in that path sensitivity is limited to a wavelength, as opposed to the Fresnel zone. As discussed by Zelt and Chen (2016), the difference arises because WDVS is based on Fermat's assumption of a stationary path, as opposed to the Born scattering assumption.

\section{Acknowledgements}

This research was funded by National Science Foundation grant EAR-1056073. We thank William Symes for providing the acoustic simulation code.

\section{References}

Biondi, B., 1997, Solving the frequency-dependent eikonal equation: in Stanford Exploration Project, Report 73, 325-338.

Brenders, A.J., and Pratt, R.G., 2007, Full waveform tomography for lithospheric imaging: results from a blind test in a realistic crustal model: Geophysical Journal International, 168, 133-151.

Chen, J., Yarman, C.E., and Cheng, X., 2014, Evaluating the performance of band-limited ray tracing through simple and complex synthetic models: in 76th EAGE Conference and Exhibition.

Chen, J., and Zelt, C.A., 2016, Application of frequencydependent traveltime tomography and full waveform inversion to realistic near-surface seismic refraction data: Journal of Environmental and Engineering Geophysics, 21, $1-12$.

Chen, J., Zelt, C.A., and Jaiswal, P., 2016, Detecting a known near-surface target through application of frequencydependent traveltime tomography and full waveform inversion to $\mathrm{P}$ - and $\mathrm{SH}$-wave seismic refraction data: Geophysics, 82, R1-R17.
Gao, F., Levander, A., Pratt, R.G., Zelt, C.A., and Fradelizio, G.L., 2007, Waveform tomography at a groundwater contamination site: Surface reflection data: Geophysics, 72, G45-G55.

Gray, S.H., and May, W.P., 1994, Kirchhoff migration using eikonal equation traveltimes: Geophysics, 59, 810-817.

Hogan, C.M., and Margrave, G.F., 2007, Ray-tracing and eikonal solutions for low-frequency wavefields: in CREWES Research Report, Vol. 19.

Hole, J.A., and Zelt, B.C., 1995, 3-D finite-difference reflection traveltimes: Geophysical Journal International, 121, 427434.

Julian, B.R., and Gubbins, D., 1977, Three-dimensional seismic ray tracing: Journal of Geophysics, 43, 95-114.

Kiser, E., Palomeras, I., Levander, A., Zelt, C., Harder, S., Schmandt, B., Hansen, S., Creager, K., Ulberg, C., Malone, S. and Moran, S., 2016, Magma reservoirs from the upper crust to the Moho inferred from high-resolution $\mathrm{Vp}$ and $\mathrm{Vs}$ models beneath Mount St. Helens: Geology, 44, 411-414.

Lomax, A., 1994, The wavelength-smoothing method for approximating broad-band wave propagation through complicated velocity structures: Geophysical Journal International, 117, 313-334.

Protasov, M.I., Yarman, C.E., Nichols, D., Osypov, K., and Cheng, X., 2011, Frequency-dependent raytracing through rugose interfaces: in Expanded Abstracts: 2011 Annual International Meeting, Society of Exploration Geophysicists, 2992-2996.

Spetzler, J., and Snieder, R., 2004, The Fresnel volume and transmitted waves: Geophysics, 69, 653-663.

Symes, W.W., Sun, D., and Enriquez, M., 2011, From modeling to inversion: Designing a well-adapted simulator: Geophysical Prospecting, 59, 814-833.

Um, J., and Thurber, C., 1987, A fast algorithm for two-point seismic ray tracing: Bulletin of the Seismological Society of America: 77, 972-986.

Vidale, J.E., 1988, Finite-difference calculation of travel times: Bulletin of the Seismological Society of America, 78, 2062-2076.

Vidale, J.E., 1990, Finite-difference calculation of travel times in three dimensions: Geophysics, 55, 521-526.

Washbourne, J.K., Bube, K.P., Carillo, P., and Addington, C., 2008, Wave tracing: Ray tracing for the propagation of band-limited signals: Part 2-Applications: Geophysics, 73, VE385-VE393.

Yarman, C.E., Cheng, X., Osypov, K., Nichols, D., and Protasov, M., 2013. Band-limited ray tracing: Geophysical Prospecting, 61, 1194-1205.

Zelt, C.A., and Barton, P.J., 1998, Three-dimensional seismic refraction tomography: A comparison of two methods applied to data from the Faeroe Basin: Journal of Geophysical Research: Solid Earth (1978-2012), 103, 7187-7210.

Zelt, C.A., and Chen, J., 2016, Frequency-dependent traveltime tomography for near-surface seismic refraction data: Geophysical Journal International, 207, 72-88. 